\title{
The role of MRI in the evaluation of painful hip joint (MRI of hip joint)
}

\section{Hayam Abd Elmonsif Abd Elatif Drar, Basma Abd Elmoneim Dessouky Mohammed, Zeinab Abd Elaziz Mohammed Ali}

Radiodiagnosis Department, Faculty of Medicine, Menoufia University, Egypt

\section{Email address:}

hayam_drar@yahoo.com (H. A. E. A. E. Drar), basmadessouky@yahoo.com (B. A. E. D. Mohammed)

\section{To cite this article:}

Hayam Abd Elmonsif Abd Elatif Drar, Basma Abd Elmoneim Dessouky Mohammed, Zeinab Abd Elaziz Mohammed Ali. The Role of MRI in the Evaluation of Painful Hip Joint (MRI of Hip Joint). International Journal of Medical Imaging. Vol. 2, No. 3, 2014 , pp. 77-82. doi: 10.11648/j.ijmi.20140203.16

\begin{abstract}
This study aim to evaluate the diagnostic value of MRI in assessment of painful hip joint. Background: Radiographic evaluation is crucial in patients with painful hip joint. Hip pain has different etiologies in adults and children. MRI is the method of choice in characterizing the various disorders and assessing the full extent of osseous, chondral and soft tissue abnormality of the hip joint. Methods: This prospective study included 100 patients with painful hip joint. The following MR sequences were performed to all patients: Coronal T1, T2 \& STIR WIs, axial T1\&T2WIs, axial T1WI and sagittal T1WI after contrast injection. Patient's history, local examination of the diseased hip and laboratory investigations were performed. Results: Male to female was 45 to 55, their ages ranged from 10 to 60 years with a mean age of 35 years. The MRI sensitivity were as follows $88 \%$ in avascular necrosis, $80 \%$ in osteoarthritis, $90 \%$ in migratory osteoporosis, $100 \%$ in transient synovitis, $72 \%$ in septic arthritis, $80 \%$ in Perthe's disease, $80 \%$ in tumor, $100 \%$ in inflammatory lesions $75 \%$ in slipped femoral epiphysis and $100 \%$ in stress fractures . Conclusion: MRI of the hip joint is an informative, diagnostic, and accurate for the assessment of hip pain and sufficient imaging modality for delineation of different hip joint pathology.
\end{abstract}

Keywords: MRI, Hip Joint, Hip Pain, Arthritis

\section{Introduction}

The hip is a stable, major weight-bearing joint with significant mobility. Hip pain has different etiologies in adults and children. In adults, hip pain may be caused by intraarticular disorders such as avascular necrosis, arthritis, loose bodies, labral tears; periarticular pathology such as tendonitis and bursitis, or extraarticular conditions such as referred pain from lumbar spine, sacroiliac joint and nerve entrapment syndromes ${ }^{(1)}$.

Magnetic resonance imaging (MRI) with its excellent soft tissue contrast and resolution with no operator dependence and no ionizing radiation is the imaging modality of choice for evaluation of hip joint abnormalities (2). MRI offers valuable information regarding occult bony and cartilage injury such as stress fractures, avascular necrosis, and osteoarthritis, as well as soft tissue abnormalities such as muscle tears and bursitis (3). MRI provides a useful assessment of patients in whom a femoroacetabular impingement is clinically suspected. A high resolution,non arthrographic technique can provide preoperative information regarding the presence and anatomic site of labral and cartilage abnormalities as well as MRI is an excellent method of preoerative assessment and helps guide the surgeon as to the likely site of labral and chondral abnormalities. Furthermore, MRI may enable the referring surgeon to decide preoperatively which patients may require an arthroscopic versus an open surgical technique (3).

\section{Material and Methods}

This prospective study was conducted on 100 patients with painful hip joint presenting various symptoms; 45 males and 55 females with age ranging between 10 and 60 years. All patients underwent complete history taking, local examination of the diseased hip and laboratory investigations. All patients underwent MRI examination of the hip joint. MRI examinations of the both hips were performed for all the patients using a TOSHIBA 1.5 Tesla MRI machine scanner. 


\subsection{Protocol of the MR Scan of the Hip Joint}

Patients were typically positioned supine on the examination table. The feet were internally rotated and gently immobilized with tape if necessary with slight flexion at the knees. Both hips were examined for suspected bilateral abnormalities using the body coil with a large field of view to determine the extent of the lesions and to allow for comparison between the normal and abnormal sides. The slice thicknesses were adjusted to be $4-8 \mathrm{~mm}$ and thinner slices were used when more details were required.

- Images were taken in the following sequences:

-Scout view of the hip: coronal TI WI image was performed for all cases for localization of subsequent slices of different planes and delineate the anatomy, coronal $\mathrm{T} 1$ and $\mathrm{T} 2$ weighted images, axial $\mathrm{T} 1$ and $\mathrm{T} 2$ weighted images, sagittal TI weighted images, coronal STIR weighted images and post contrast TI weighted images in sagittal, axial and coronal planes were performed immediately after intravenous bolus injection of Gadolinium DTPA in dose of $0.1 \mathrm{mmoll} / \mathrm{kg}$ in the inflammatory and neoplastic cases. The examinations were recorded on compact discs (CDs) that were meticulously revised to detect and assess any abnormalities that would justify the patients' complaint.

\section{Results}

Of the studied one hundred patients with painful hips, Male to female was 45 to 55 , their ages ranged from 10 to 60 years with a mean age of 35 years. The most frequently involved age group was the group $>30-40$.

As all the patients were presented by painful hips, additional clinical presentations were also determined. The most frequent clinical presentation following hip pain was lower back pain $(n=20,20 \%)$, followed by limited movement $(n=15,15 \%)$, hip joint swelling $(n=4,4 \%)$ and hip pain and constitutional symptoms $(n=6,6 \%)$. Based on the findings obtained by MRI, $15 \%$ of the patients had normal studies, however in the remaining $85 \%$ the final diagnoses were as the following: avascular necrosis (25\%), osteoarthritis (10\%), migratory osteoporosis (10\%), transient synovitis (10\%), septic arthritis (7\%), Perthe's disease $(5 \%)$, tumor $(5 \%)$, intramuscular inflammatory lesions $(5 \%)$, slipped femoral capital epiphysis $(4 \%)$ and stress fractures $(4 \%)$.

The diagnostic MRI findings seen in the 25 cases with avascular necrosis are described: Table (1).

Table 1. Showed the diagnostic MRI findings seen in cases with avascular necrosis.

\begin{tabular}{lll}
\hline MRI findings & No. of cases & $\%$ \\
\hline Focal subchondral signal abnormality & 25 & $100 \%$ \\
Double line sign & 20 & $80 \%$ \\
Marrow edema & 10 & $40 \%$ \\
Joint effusion & 8 & $32 \%$ \\
\hline
\end{tabular}

The diagnostic MRI findings seen in the 10 cases with osteoarthritis are described: Table (2).

Table 2. Showed the diagnostic MRI findings seen in cases with osteoarthritis.

\begin{tabular}{lll}
\hline MRI findings & No. of cases & $\%$ \\
\hline Joint effusion & 10 & $100 \%$ \\
Synovial thickening & 7 & $70 \%$ \\
Marrow edema & 7 & $70 \%$ \\
Subchondral cysts & 5 & $50 \%$ \\
Joint space narrowing & 4 & $40 \%$ \\
Marginal osteophytes & 4 & $40 \%$ \\
Soft tissue edema & 3 & $30 \%$ \\
\hline
\end{tabular}

The diagnostic MRI findings seen in the 10 cases with migratory osteoporosis are described: Table (3).

Table 3. The diagnostic MRI findings seen in cases with migratory osteoporosis.

\begin{tabular}{lll}
\hline MM MRI findings & No. of cases & $\%$ \\
\hline Marrow edema of femoral head and neck & 10 & $100 \%$ \\
Joint effusion & 5 & $50 \%$ \\
Marrow edema of the acetabulam & 4 & $40 \%$ \\
\hline
\end{tabular}

The sensitivity of the MRI in the diagnosis of different hip joint lesions according to the true positive and false negative cases is described: Table (4).

Table 4. Showed the sensitivity of the MRI in the diagnosis of different hip joint lesions.

\begin{tabular}{|c|c|c|c|c|}
\hline Final diagnosis & $\begin{array}{l}\text { No. of } \\
\text { patients }\end{array}$ & $\begin{array}{l}\text { True } \\
+ \text { ve }\end{array}$ & $\begin{array}{l}\text { False - } \\
\text { ve }\end{array}$ & Sensitivity \\
\hline Avascular necrosis & 25 & 22 & 3 & $88 \%$ \\
\hline Osteoarthritis & 10 & 8 & 2 & $80 \%$ \\
\hline $\begin{array}{l}\text { Migratory } \\
\text { osteoporosis }\end{array}$ & 10 & 9 & 1 & $90 \%$ \\
\hline Transient synovitis & 10 & 10 & 0 & $100 \%$ \\
\hline Septic arthritis & 7 & 5 & 2 & $72 \%$ \\
\hline Perthe's disease & 5 & 4 & 1 & $80 \%$ \\
\hline Extraarticular tumor & 5 & 4 & 1 & $80 \%$ \\
\hline $\begin{array}{l}\text { Intramuscular } \\
\text { inflammatory lesions }\end{array}$ & 5 & 5 & 0 & $100 \%$ \\
\hline $\begin{array}{l}\text { Slipped femoral } \\
\text { capital epiphysis }\end{array}$ & 4 & 3 & 1 & $75 \%$ \\
\hline Stress fractures & 4 & 4 & 0 & $100 \%$ \\
\hline
\end{tabular}

\section{Discussion}

MRI is the most sensitive mean of diagnosing AVN, representing the gold-standard of noninvasive diagnostic evaluation. It has several advantages, allows accurate 
staging by clearly depicting the size of the lesion, it also detects asymptomatic lesions that are undetectable on plain radiographs, thus facilitating early treatment and better response. It provides multiplanar imaging and excellent soft tissue resolution and can demonstrate response of the femoral head to treatment ${ }^{(4)}$

In this study, in patients with avascular necrosis, the disease was bilateral in (8) $32 \%$ of patients and unilateral in the remaining (17) $68 \%$ of patients, 4 patients of these were post traumatic sequel. Kim et al ${ }^{(5)}$ reported that trauma involving the proximal femur or acetabulum may lead to unilateral avascular necrosis however, non-traumatic avascular necrosis, is bilateral in $50-80 \%$ of patients.

Takatori Y et al ${ }^{(6)}$ reported that $85 \%$ of the patients of avascular necrosis show a characteristic "double line" sign on T2 weighted images which is a specific finding that consists of concentric low- and high signal intensity rims that surround the area of marrow signal intensity change within the femoral head.

In this study, the characteristic "double line" sign on T2 weighted images was seen in $80 \%$ of patients with AVN (20 patient). The MRI findings were true positive in 22 patients, false negative in 3 patients and the sensitivity of MRI in diagnosis of avascular necrosis was $88 \%$.

MR imaging is reported to have a sensitivity of $95 \%$ and specificity of $98 \%$ in the diagnosis of AVN of the hip. It detects the bone marrow edema associated with early AVN, which is seen as decreased signal intensity with poorly defined margins on T1-weighted images ${ }^{(6)}$.

Hayashi (7) reviewed that bone marrow signal alteration (bone marrow edema) is a common magnetic resonance imaging feature of hip OA and the degree of bone marrow edema, as assessed by MRI, correlates with the severity of hip pain in cases with osteoarthritis .

In this study, the 10 patients diagnosed as osteoarthritis, the disease was bilateral in $40 \%$ of cases (4 patients) and unilateral in the remaining $60 \%$ of patients (6 patients). Bone marrow edema was noted in $70 \%$ of patients ( 7 patients).

MRI is the imaging modality of choice, demonstrating diffuse low signal on $T_{1}$ weighted images and high signal on $\mathrm{T}_{2}$ fat suppressed or STIR images, indicating bone marrow edema, several weeks before radiographic changes are present ${ }^{(8)}$.

In this study 10 patients were diagnosed as migratory osteoporosis, MRI findings were true positive in 9 patients. The diagnosis was confirmed by clinical and follow up MRI scan after conservative treatment and the disease was self limited.

Yang et al ${ }^{(9)}$ reported that the that the main diagnostic MRI findings seen in transient synovitis are joint effusion, presence of synovial thickening, alterations in signal intensity of soft tissue and bone marrow in the affected hip joint.
In this study 10 patients were diagnosed as transient synovitis and the dominant MRI finding was joint effusion which was identified in all the $10(100 \%)$ patients, followed by synovial thickening was identified in $7(70 \%)$ patients, enhanced synovium in $5(50 \%)$ patients and marrow edema in $2(20 \%)$ patients.

Most cases of LCP disease are unilateral, approximately $15 \%$ of individuals are affected bilaterally . When bilateral, the femoral heads are most commonly asynchronously affected. LCP disease is generally considered to be idiopathic without an identifiable inciting factor ${ }^{(10)}$.

In this study, the 5 patients with Legg-Calvé-Perthes disease were unilateral in $4(80 \%)$ of patients and bilateral in the remaining one $(20 \%)$ of patients.

Dillon (11) reported that MRI provides an early and sensitive method for detecting the pre-slip stage of the proximal femoral epiphysis and the earliest evidence of slipped capital femoral epiphysis on MRI is diffuse or globular epiphyseal widening and hyperintense signal of the bone marrow along the epiphysis on T2-weighted images which indicate stress and edema.

In this study the 4 patients diagnosed as slipped femoral capital epiphysis, The dominant MRI finding was femoral epiphysis widening and displacement which was identified in all the $4(100 \%)$ patients, followed by focal subchondral signal abnormality that was identified in $3(75 \%)$ patients, bone marrow edema in $3(75 \%)$ patients and joint effusion in $2(50 \%)$ patients.

Deutsch et al. ${ }^{(12)}$ reported that magnetic resonance imaging with limited T1-weighted coronal images is $100 \%$ accurate in detecting occult hip fractures. When MRI and clinical outcome were used as the standard of reference in a study included 50 patient, the prospective accuracy of MRI in the diagnosis of the presence or absence of hip fracture was $100 \%$.

In this study 4 patients diagnosed as stress fractures, MRI findings were true positive in the 4 patients and the dominant MRI finding in patients with stress fractures was bone marrow edema that was identified in the $4(100 \%)$ patients, followed by femoral head subchondral linear hypointense line in the $3(75 \%)$ patients and femoral neck linear hypointensity in $1(25 \%)$ patients. So the sensitivity of MRI in diagnosis of stress fractures was $100 \%$.

Magnetic resonance imaging facilitates the early diagnosis of stress fracture as it is presents with bone marrow edema best depicted on fat-suppressed T2weighted scans or short tau inversion recovery (STIR) images, the increased water content of the associated medullary edema or hemorrhage results in high-signal intensity against the dark background of suppressed fat. The fracture line itself may not always be visible as a discrete low signal intensity line, instead, only an amorphous region of bone marrow edema may be identified (13) . 


\section{Conclusion}

MRI is the method of choice in characterizing the various disorders and assessing the full extent of osseous, chondral and soft tissue involvement. MR imaging can also accurately demonstrate joint effusions, synovial proliferations, articular cartilage abnormalities, subchondral bone, ligaments, muscles and juxta articular soft tissues. The MRI gave accurate diagnosis in $88 \%$ of patients with avascular necrosis, $80 \%$ of patients with osteoarthritis, $90 \%$ of patients with migratory osteoporosis, $100 \%$ of patients with transient synovitis, $72 \%$ of patients with septic arthritis, $80 \%$ of patients with Perthe's disease,
$80 \%$ of patients with extraarticular tumor, $100 \%$ of patients with intramuscular inflammatory lesions, $75 \%$ of patients with slipped femoral capital epiphysis and $100 \%$ of patients with Stress fractures. In this study we reached that MRI is the method of choice in characterizing the various disorders of the hip joint and accurate imaging modality for assessing the full extent of osseous, chondral and soft tissue abnormality of the hip joint.

Finally we conclude that MRI of the hip joint is an informative, diagnostic, non invasive, rapid and accurate imaging modality for the assessment of hip pain and sufficient imaging modality for delineation of different hip joint pathology.
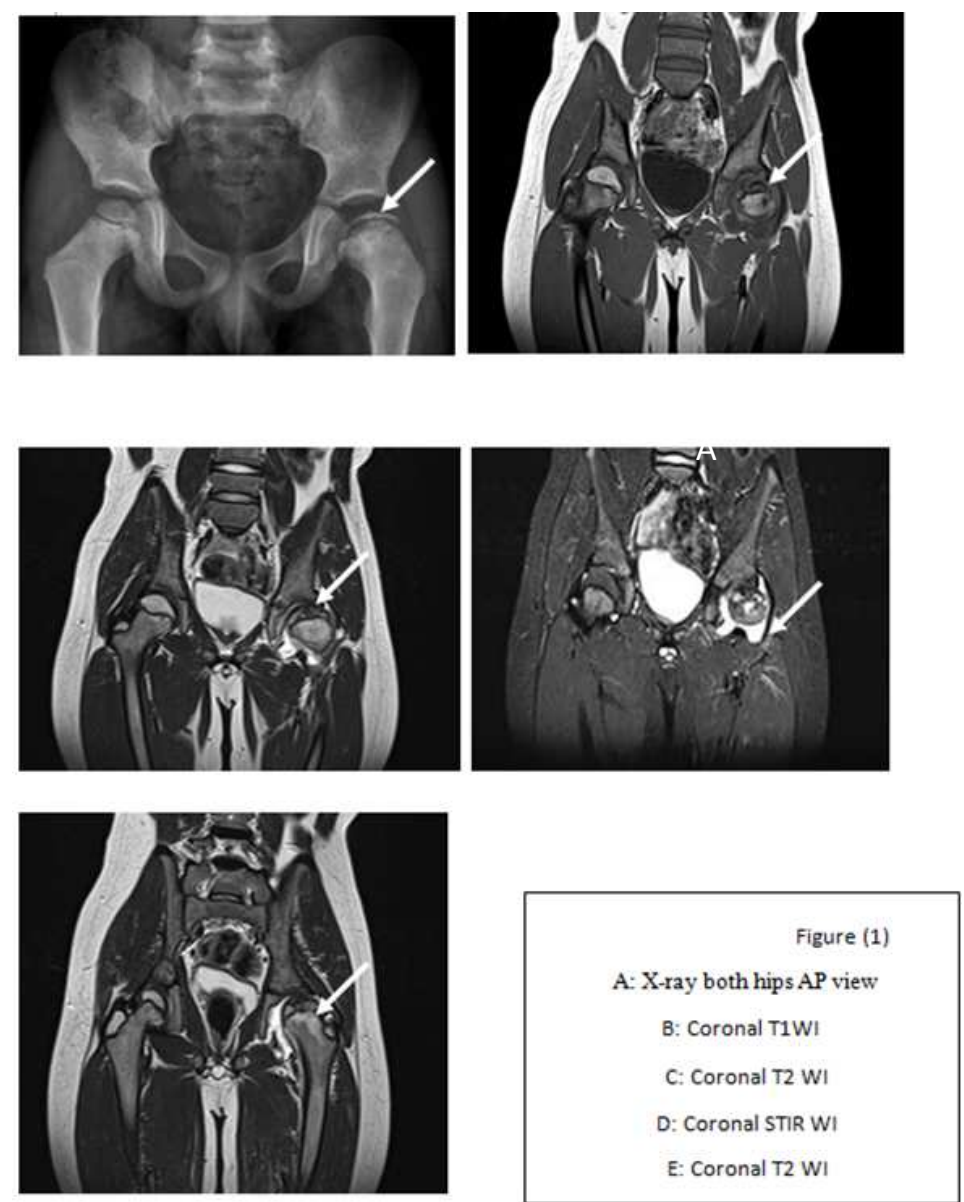

Fig 1. Case No. 1. Irregularity and flattening of the left femoral epiphysis with irregular foci of abnormal signal on T1 WI \&T2WI Perthe's disease with mild effusion. 

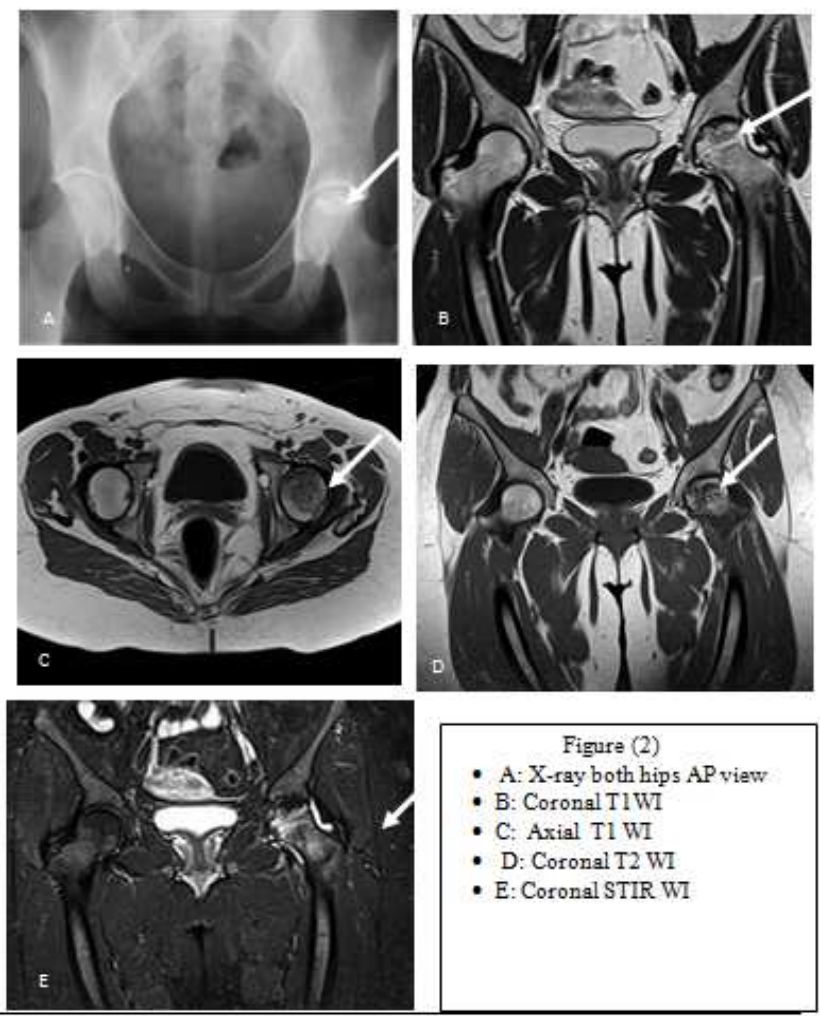

Figure (2)

- A: X-ray both hips AP view

- B: Coronal T1W

- C: Axial Tl WI

- D: Coronal T2 WI

- E: Coronal STIR WI

Fig 2. Case No. 2. Sclerosis of the left femoral epiphysis with subchondral fissures, "double-line sign" and altered marrow signal Grade 3 avascular necrosis with mild joint effusion.
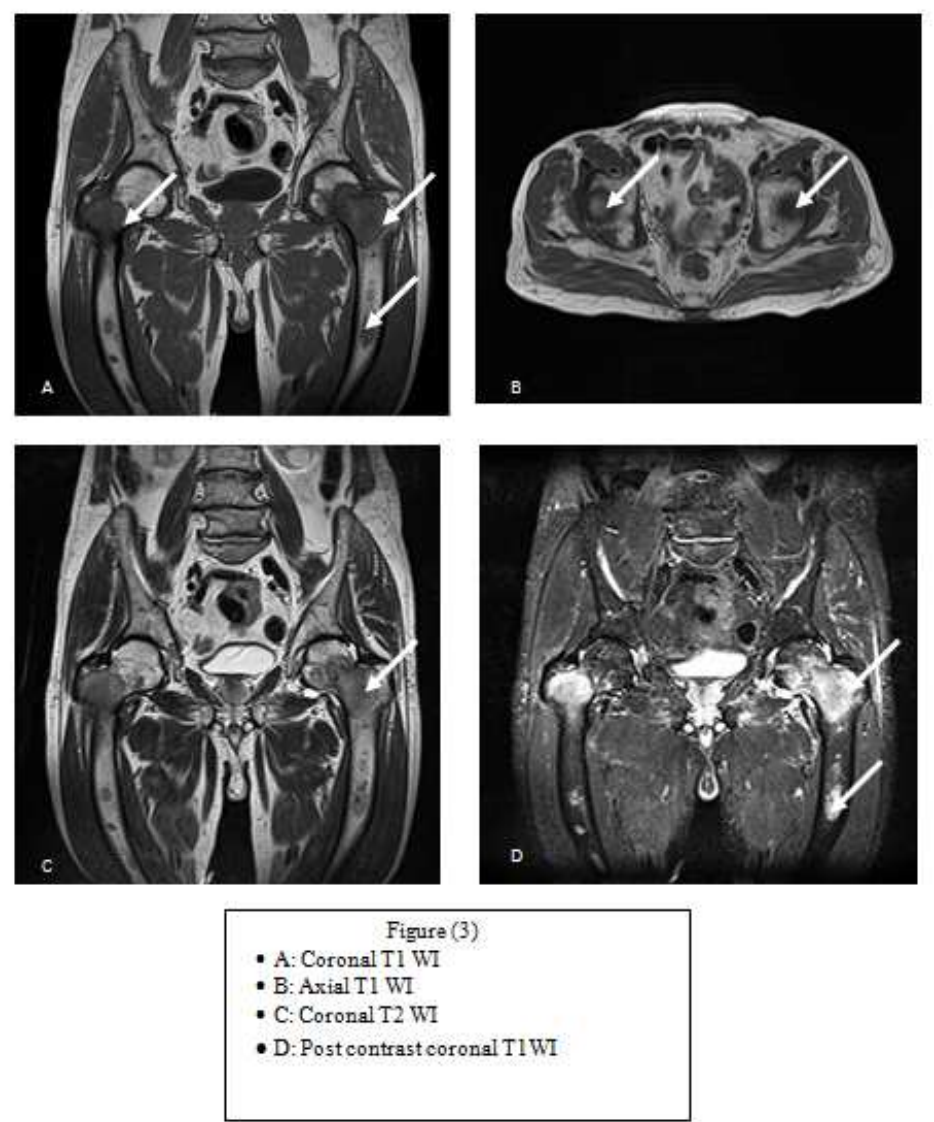

Fig 3. Case No. 3: Multiple marrow infiltrative lesions are seen involving all of the visualized bones, hypointense in T1WI \& mixed in T2WI with moderate enhancement Wide spread osseous deposits. 

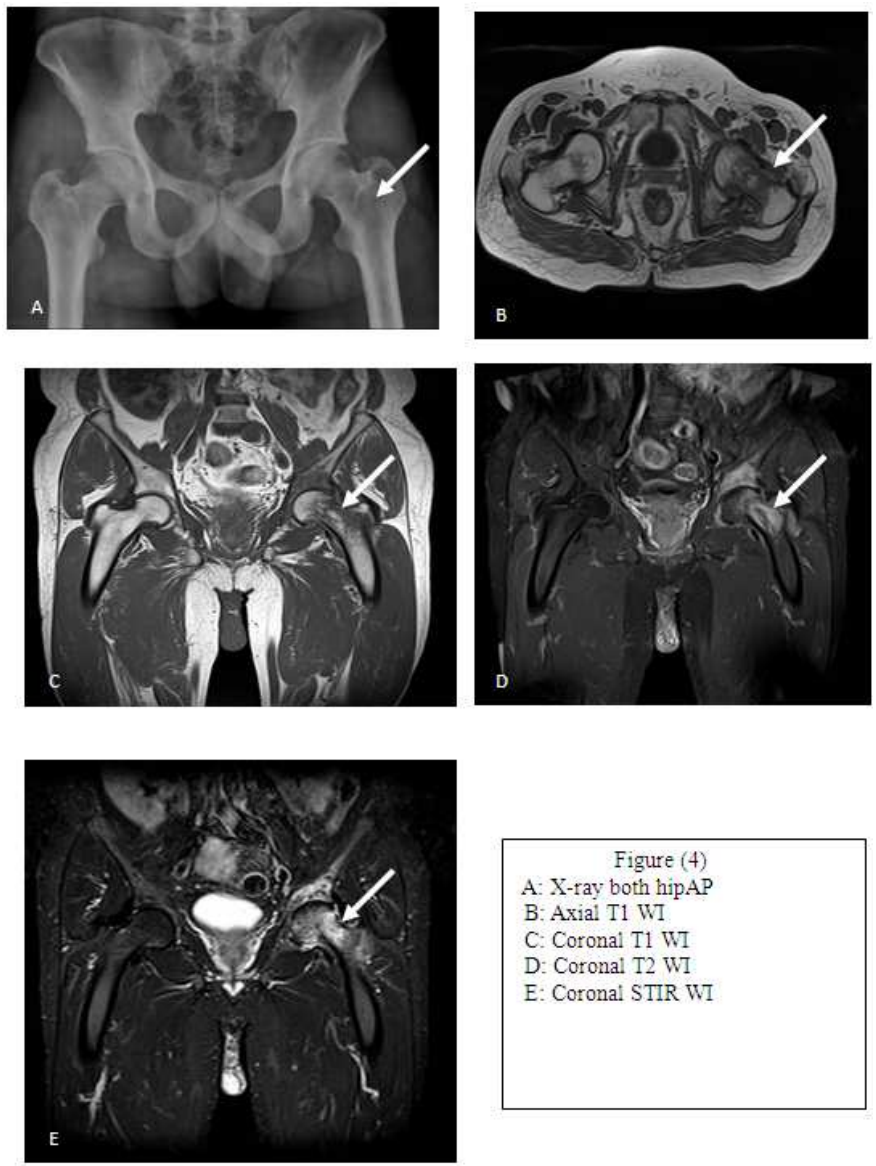

Fig 4. Case No. 4: Diffuse abnormal signal within the left femoral head and neck and the acetabulum, homogeneously hypointense in T1 \&hyperintense in T2 \&STIR Migratory osteoporosis.

\section{References}

[1] Ghebontni L, El-khoury J, Grenier PA. MR imaging of the hip: Normal intra-articular structures and common disorders. Eur Radiol 2009; 10:83-8.

[2] Boutry N, Fredoux D, Migaud H. Rapidly destructive osteoarthritis of the hip: MR imaging findings. AJR Am J Roentgenol 2005; 179: 657-3.

[3] Verbeeten KM, Hermann KL, Hasselqvist M. The advantages of MRI in the detection of occult hip frar.ures. Eur Radiol 2007; 15:165-7.

[4] Aldridge JM, Urbaniak JR. Avascular necrosis of the femoralhead: etiology, pathophysiology,classification, and current treatmentguidelines. Am J Orthop Jul 2008; 33:327-2.

[5] Kim YM, Kim HJ. The pattern of bone marrow edema on MRI in osteonecrosis of the femoral head. J Bone Joint Surg Br 2007; 82:837-1.

[6] Takatori Y, Kokubo T, Ninomiya S. Avascular necrosis of the femoral head. JBJS 2000; 75: 217-1.

[7] Hayashi D, Roemer FW, Felson DT. Magnetic resonance imaging of subchondral bone marrow lesions in association with osteoarthritis. Semin Arthritis Rheum 2012; 42: 105-8.

[8] Vande BC, Malghem JJ, Lecouvet FE. Idiopathic bone marrow edema lesions of the femoral head: predictive value of MR imaging findings. Radiology 2003; 212:527-5.

[9] Yang WJ, Lim GY. MR imaging of transient synovitis: differentiation from septic arthritis. Pediatr Radio 2009; 1 39:532-7.

[10] Ducou L, Pointe H, Haddad S. Legg-Perthes-Calvé disease: staging by MRI using gadolinium. Pediatr Radiol 2000; 24 : 88-1.

[11] Dillon JE, Connolly SA, Connolly LP. MR Imaging of Congenital /Developmental and Acquired Disorders of the Pediatric Hip and Pelvis. Magnetic Resonance Imaging Clinics of North America 2009; 13: 55-9.

[12] Deutsch A., Mink L. and Waxman A. Occult fractures of the proximal femur: MR imaging. Radiology, 2005;170:113116.

[13] Quinn S. and Carthy J. Prospective evaluation of patients with suspected hip fracture and indeterminate radiographs: Use of T1 weighted MR images. 2002; Radiology, 187:469471. 